\title{
Eficiência técnica na suinocultura: Efeitos dos gastos com meio ambiente e da renúncia fiscal
}

\author{
Benedito D. Pereira ${ }^{1}$, João C. de S. Maia ${ }^{2} \&$ Rosalina Camilot ${ }^{3}$
}

\begin{abstract}
RESUMO
A criação de suínos em escala industrial resulta em intensa produção de dejetos nas propriedades rurais, conseqüências que se manifestam no solo, no ar, na fauna, na flora e no ambiente socioeconômico; neste contexto, externalidades negativas podem interagir com outras variáveis ou ações econômicas representadas, por exemplo, pelos gastos com a conservação do meio ambiente e, em particular, em Mato Grosso, pela participação no Programa Granja de Qualidade, um instrumento estadual de renúncia fiscal. Depois de estimados os índices de eficiência técnica com dados de dez suinoculturas situadas em Mato Grosso a partir de função de produção, cuja variável dependente é representada pela produção de leitões e as independentes, pelo consumo de água, de eletricidade e da quantidade de trabalhadores diretamente vinculados ao processo produtivo, verifica-se que os gastos com a conservação do meio ambiente exercem efeitos positivos sobre os índices de eficiência técnica, enquanto a participação no Programa Granja de Qualidade, diferentemente da variável anterior, se revela estatisticamente não significativa.
\end{abstract}

Palavras-chave: externalidades, conservação, política fiscal

\section{Technical efficiency in swine breeding: Effect of the expenses with the environment and the fiscal renunciation}

\begin{abstract}
Swine creation on an industrial scale results in intense production of dejections in the rural properties, consequences that reveal in the soil, air, fauna, flora and socio-economic environment; in this context, negative externalities can interact with other variable or economic actions, represented, for example, by the expenses with the conservation of the environment and, in particular, in Mato Grosso, by participation in the Program of Quality Farm, a state instrument of fiscal renunciation. After estimating the technical efficiency indices with data of ten properties situated in Mato Grosso from production functions, whose dependent variable is represented by the production of swine and the independent ones are the consumption of water, electricity and the amount of workers directly tied with the productive process. It was verified that the expenses with the conservation of the environment exert a positive effect on technical efficiency indices, while the participation in the Program of Quality Farm, differently from the previous variable, shows itself to be statistically not significant.
\end{abstract}

Key-words: externalities, conservation, fiscal policy

${ }^{1}$ DE/UFMT, Av. Fernando C. da Costa, s/n, CEP 78060-900, Cuiabá, MT. Fone: (65) 3615-8515. E-mail: bdp@terra.com.br

${ }^{2}$ DA/UFMT. Fone: (65) 3615-8643. E-mail: jotace@terra.com.br

${ }^{3}$ Mestre pelo Programa de Agricultura Tropical (UFMT). Fone: (65) 3615-8643. E-mail: rosalina.camilot@gmail.com 


\section{INTRODUÇÃO}

A economia de Mato Grosso vem exibindo expressivo crescimento do seu Produto Interno Bruto (PIB) nos últimos anos, que tem sido maior que o do país como um todo. Entre 1985 e 2003, de acordo com a SEPLAN-MT (2004), dentre as unidades federativas do Brasil, liderado pela produção agropecuária o PIB mato-grossense foi o que exibiu maior expansão nominal: 275\%. Estatísticas e análises mais detalhadas desta economia constam em Pereira (2007). A suinocultura é uma das atividades que se têm destacado na agropecuária mato-grossense; como ilustração desse fato ressalta-se que o efetivo, em 2000, do rebanho de suínos no Estado, de 834.084 cabeças, no ano seguinte cresceu para 1.114.592 cabeças; assim, nesses dois anos o aumento da população foi de 33,63\%.

Esta franca expansão pode ser explicada pelas condições competitivas do estado, como grande produtor de grãos, presença de clima favorável, água em abundância, o fato de Mato Grosso se constituir em área livre de aftosa (com vacinação), sua inclusão como área livre de peste suína clássica e, ainda, a partir de 1995, a existência do Programa Granja de Qualidade, que viabiliza a concessão de incentivo fiscal (renúncia de 66,66\%) do Imposto sobre Circulação de Mercadorias e Serviços (ICMS) incidente sobre a comercialização de animais. Por oportuno, em Mato Grosso o número de suínos que participa deste programa aumentou de 41.850 em 1995, para 541.609, em 2002; por outro lado, a proporção do número de suínos que participa do programa em relação ao rebanho total de suínos no estado, nesses dois anos, expandiu de 4,65 para 52,35\%, apontando que ele tem despertado elevado interesse na suinocultura do estado. Para obter o incentivo fiscal, o criador deve cumprir algumas exigências: contar com assistência técnica especializada, fazer reaproveitamento dos dejetos produzidos na granja, obedecer às normas de higiene e de sanidade específicas e manter registros produtivos à disposição da administração do programa.

De modo geral, o crescimento da suinocultura causa diversos efeitos na socioeconomia e no meio ambiente físico (solo, água, ar, fauna e flora); esses resultados podem ser positivos ou adversos, ou seja, eles podem gerar custos ou benefícios; se os custos superam os benefícios, esta atividade pode comprometer importantes indicadores de sustentabilidade da economia do estado.

De forma especial, os efeitos da suinocultura (produção de dejetos) sobre o solo ocorrem em função da quantidade de esterco líquido que neste pode ser depositado, alterando sua capacidade de filtração e retenção de nutrientes; além disso, seus efeitos sobre a água são provocados sobretudo, pelo nitrogênio e excesso de fósforo expelidos pelo processo produtivo suinícola, cujo efeito sobre o ar se dá em decorrência da emissão de gases tóxicos, poluentes e odores desagradáveis ao bem-estar humano; por sua vez, as influências da atividade em questão sobre a fauna e a flora decorrem, fundamentalmente, devido aos resultados que esta atividade provoca e que contribuem para o desaparecimento de determinadas espécies de animais e vegetais.
Em Mato Grosso, diversas pesquisas vêm sendo realizadas abordando questões ambientais relacionadas à suinocultura, citando-se, como exemplo, Alves (2001), que identifica e estuda diversas tecnologias que estão sendo empreendidas na atividade suinícola; Freitas (2004), que estima (valora) e busca a compreensão do valor de existência de ativos ambientais impactados pela criação de suínos na principal granja localizada em Mato Grosso, e Anunciato (2004), que estima indicadores de estado em diversas granjas suinícolas localizadas em vários municípios matogrossenses. Esses e outros estudos são explicados pela crescente importância que as questões ambientais vêm exibindo nos últimos anos, insertas nas discussões e reflexões sobre o desenvolvimento sustentável.

Resultante de pesquisas em empresas suinícolas situadas em municípios de Mato Grosso, o objetivo deste artigo é, inicialmente, estimar índices de eficiência técnica a partir de função de produção a ser obtida; após isto, procura-se identificar fatores explicativos desses índices, averiguandose se gastos com conservação do meio ambiente e participação no Programa Granja de Qualidade, exercem ou não efeitos positivos sobre os índices de eficiência técnica.

\section{MATERIAL E MÉTODOS}

Amplamente adotada em pesquisas aplicadas e fundamentada em diversos pressupostos, a análise de regressão se constitui em modelo econométrico voltado à identificação e mensuração de relações causais entre variáveis, usado neste artigo em duas regressões; na primeira, estima-se a função de produção por intermédio do método de mínimos quadrados ordinários; com tal estimativa, são obtidos os índices de eficiência técnica; na segunda, identificamse variáveis explicativas desses índices.

Para se estimar a eficiência técnica, recordando-se, previamente, necessita-se estimar uma função de produção; aqui, esta função tem como variável dependente a produção anual de leitões das empresas investigadas e, como variáveis independentes: o número de trabalhadores do processo produtivo, gastos com energia elétrica e o consumo de água; tal função pode ser determinística ou estocástica: se determinística, ela define a relação exata entre as quantidades de insumo que o empresário emprega e a quantidade produzida; se, porém, estocástica, a função de produção incorpora componente de aleatoriedade dado que, sob esta perspectiva, ela também depende do distúrbio estocástico da função econométrica que lhe está associada.

Neste caso, ou seja, incorporando-se componente de aleatoriedade, a função estimada pode ser considerada estocástica de fronteira com erro composto e estocástica de fronteira full, conforme abordam Souza Júnior et al. (2005), Magalhães \& Campos (2006) e Mariano \& Sampaio (2002); na função estocástica de fronteira com erro composto, o erro ou distúrbio aleatório $(\varepsilon)$ pode ser dividido em duas partes: atribuída a erros estatísticos, uma capta os efeitos dos erros de mensuração, das falhas estatísticas e dos fatores externos ao controle da unidade produtiva, como mudança de clima 
e outras causas imprevisíveis, a outra parte de e, tomandose como referência os desvios do produto observado em relação ao potencial, estima ineficiência técnica; a primeira parte de $e$ é composta de uma variável aleatória simétrica enquanto a outra, que mede a eficiência técnica, se constitui em variável unilateral.

Contemplando fronteira de produção estocástica com erro unilateral, Timmer (1971) sugeriu método de estimação dos índices de eficiência técnica de maneira simples e direta: calculando-se a razão entre o produto observado e o potencial, ou seja, ET = (expu) $=\mathrm{Y}($ observado $) / \mathrm{Y}($ potencial $)$, como citam Mariano (1999) e Pereira \& Mendes (2002); mais detalhadamente, de acordo com esse método o índice de eficiência técnica de cada propriedade é calculado por intermédio da razão entre o produto observado e o produto potencial; após isto, para se mudar a função estimada de maneira que nenhum resíduo seja positivo, o intercepto da função é corrigido por intermédio do maior resíduo estimado; o produto observado é representado pela produção da unidade suinícola, colhida na pesquisa de campo; por sua vez, o produto potencial se constitui na produção estimada através da função de produção antes comentada; neste artigo, para se obter a eficiência técnica das unidades investigadas adota-se o método sugerido por Timmer (1971).

Para se estimar a primeira regressão linear múltipla foram testadas várias formas funcionais, em que a linear se revelou a mais indicada; adotando-se esta forma funcional, tem-se a seguinte especificação: $L=\beta_{0}+\beta_{1} \mathrm{~T}+\beta_{2} \mathrm{EE}+\beta_{3} \mathrm{~A}$ $+e$ (1), donde $\beta_{0}, \beta_{1}, \beta_{2}$ e $\beta_{3}$ denotam os parâmetros a serem estimados, enquanto $e$ indica o componente residual que estima a ineficiência técnica; neste modelo, a variável dependente (L) é representada pela produção anual de leitões obtida pelas empresas investigadas; as variáveis independentes são: número de trabalhadores do processo produtivo (T), gastos com energia elétrica (EE) (quantidade de KWh utilizada pela empresa no ano de referência, convertida em R\$) e consumo de água (A) (consumo anual em $\mathrm{m}^{3}$ de água no processo produtivo); esta primeira regressão tem como objetivo estimar os índices de eficiência técnica da forma anteriormente mencionada.

Para depois se verificar se as variáveis independentes selecionadas exercem ou não efeitos estatisticamente significativos sobre os índices de eficiência técnica, obtidos na primeira regressão, recorre-se mais uma vez à análise de regressão adotando-se novamente o método dos mínimos quadrados ordinários; caso em que se tem a seguinte especificação: $\mathrm{ET}=\beta_{0}+\beta_{1} \mathrm{GMA}+\beta_{2} \mathrm{GQ}+e$ (2), donde $\beta_{0}, \beta_{1}$ e $\beta_{2}$ são os coeficientes a serem estimados e $e$ indica o resíduo aleatório; também aqui a regressão linear resultou preferida quando comparada com outras formas funcionais; em resumo, nesta regressão a variável dependente é o vetor constituído pelos índices de eficiência técnica (ET) da função de produção enquanto as variáveis independentes são os gastos com o meio ambiente (GMA), além de uma variável dummy (GQ), que assume o valor um, quando a empresa participa do Programa Granja de Qualidade, e valor zero, em caso contrário. Os gastos com a conservação do meio ambiente contemplam as despesas incorridas com a conservação dos ativos ambientais (solo, água, ar, fauna e flora), canalizadas essencialmente para a manutenção da lagoa de decantação dos dejetos do processo produtivo da suinocultura.

\section{RESULTADOS E DISCUSSÃO}

Os dados da pesquisa, colhidos em 2004 e anotados na Tabela 1, foram coletados em dez granjas suinícolas situadas nos seguintes municípios mato-grossenses: Rondonópolis, Pedra Preta, Sorriso, Nova Mutum e Campo Verde.

Tabela 1. Quantidade de leitões produzidos por ano (L), número de trabalhadores do processo produtivo $(\mathrm{T})$, gasto anual com energia elétrica (EE) e consumo anual de água (A)

\begin{tabular}{|c|c|c|c|c|}
\hline Observação & L (unidade) & $\mathrm{T}$ (unidade) & EE (R\$) & $A\left(m^{3}\right)$ \\
\hline 1 & 600 & 2 & 1.920 & 2.520 \\
\hline 2 & 2.400 & 4 & 6.000 & 6.800 \\
\hline 3 & 4.320 & 5 & 5.760 & 9.400 \\
\hline 4 & 30.000 & 27 & 38.039 & 61.000 \\
\hline 5 & 25.794 & 25 & 34.000 & 45.000 \\
\hline 6 & 19.200 & 15 & 25.100 & 20.800 \\
\hline 7 & 16.800 & 10 & 22.100 & 21.000 \\
\hline 8 & 35.000 & 33 & 40.000 & 48.000 \\
\hline 9 & 640 & 2 & 1.800 & 2.400 \\
\hline 10 & 15.600 & 12 & 22.200 & 24.000 \\
\hline
\end{tabular}

Os resultados da primeira regressão, em que o “*” indica que o parâmetro é significativo a nível de significância de 5\%, foram:

$$
\begin{array}{rllll}
\mathrm{L}=-1,49+460,59 \mathrm{~T}+0,65 \mathrm{EE}-0,10 \mathrm{~A} & \mathrm{R}^{2}=0,9938 & \mathrm{~F}=324,44 \\
(-2,2) & (2,90)^{*} & (5,65)^{*}(-1,42) & \mathrm{d}=1,91 & \mathrm{n}=10
\end{array}
$$

$\mathrm{O}$ valor de $\mathrm{R}^{2}$ obtido explana quase a totalidade das variações da variável dependente enquanto o valor de F, estatisticamente significativo, revela que o conjunto das variáveis independentes explica as variações da variável dependente, com nível de significância de 5\%; verificou-se, em adição, que duas das possíveis violações dos pressupostos básicos da regressão múltipla, a multicolinearidade e a heterocedasticidade, não são estatisticamente significativas; em seguida e como o teste de Durbin-Watson revelou ser inconcluso para a detecção de autocorrelação dos resíduos, recorreu-se ao teste de Godfrey, que apontou a não-existência de autocorrelação serial. Ademais, por intermédio do teste de Jarque-Bera, notou-se que os resíduos exibem distribuição normal, não obstante o relativamente reduzido número de observações da pesquisa; por sua vez, também com nível de significância de 5\%, os valores da estatística t mostram que o coeficiente da variável T e o da EE são estatisticamente significativos, enquanto o coeficiente da variável A, ao contrário dessas duas variáveis, é desprovido de significância estatística; em síntese, o número de trabalhadores e os gastos com energia elétrica, diferentemente do consumo de água, exercem efeitos, nesses casos positivos, sobre a quantidade produzida de leitões. 
Diante do exposto e como já explanado, após se substituírem os valores observados de T, EE e A da Tabela 1 na equação estimada (Eq. 1), obtiveram-se os valores estimados da produção de leitões, os quais estão anotados na Tabela 2; através desse método se estimam os parâmetros da função fronteira e se obtêm estimativas não enviesadas dos coeficientes dessa função após o que, para que nenhum resíduo seja positivo, o intercepto da função foi corrigido por intermédio do maior resíduo estimado.

Tabela 2. Estimativa da produção de leitões $\left(\mathrm{L}_{\mathrm{Ee}}\right)$ (produto potencial estimado) (1.000 unidades)

\begin{tabular}{lcccccccccc}
\hline Observação & $\mathbf{1}$ & $\mathbf{2}$ & $\mathbf{3}$ & $\mathbf{4}$ & $\mathbf{5}$ & $\mathbf{6}$ & $\mathbf{7}$ & $\mathbf{8}$ & $\mathbf{9}$ & $\mathbf{1 0}$ \\
Valores Estimados $\left(\mathrm{L}_{\mathrm{Ee}}\right)$ & 2 & 5 & 5 & 31 & 29 & 21 & 17 & 36 & 2 & 17 \\
\hline
\end{tabular}

Os índices de eficiência técnica de cada propriedade, situados entre zero e um e que estão anotados na Tabela 3, relembrando-se, foram obtidos pela razão entre o produto observado (Tabela 1) e o produto potencial estimado (Tabela 2); utilizando-se como exemplo o valor da observação 1 , o índice 0,31 aponta que a empresa em questão consegue produzir, em média, 31\% da produção alcançada pela firma mais eficiente tecnicamente; já para a observação 7 , também como ilustração, o valor 0,99 traduz que a empresa consegue produzir, em média, 99\% da produção alcançada pela firma mais eficiente.

Tabela 3. Índices de Eficiência Técnica (ET), gastos com meio ambiente (GMA) e participação ou não no Programa Granja de Qualidade (GQ)

\begin{tabular}{ccrc}
\hline Observação & ET & GMA (R\$) & $\begin{array}{l}\text { Participação no Programa } \\
\text { Granja de Qualidade (1, se } \\
\text { participa e 0, se não) }\end{array}$ \\
1 & 0,31 & 1.000 & 0 \\
2 & 0,47 & 2.880 & 0 \\
3 & 0,84 & 4.800 & 0 \\
4 & 0,96 & 34.000 & 1 \\
5 & 0,88 & 30.000 & 1 \\
6 & 0,90 & 30.000 & 0 \\
7 & 0,99 & 25.000 & 0 \\
8 & 0,98 & 32.000 & 0 \\
9 & 0,34 & 1.200 & 0 \\
10 & 0,89 & 18.200 & 0 \\
\hline
\end{tabular}

Adotando-se como variável dependente o vetor com os índices de eficiência técnica e como variáveis independentes, os gastos com conservação do meio ambiente (GMA) e participação ou não no Programa Granja de Qualidade (GQ), cujos valores constam na Tabela 3 neste estágio do trabalho, realizouse uma segunda regressão. É natural que, dentre as unidades investigadas, a existência de apenas duas unidades com participação no Programa Granja de Qualidade, restringe os resultados da regressão sem, contudo, comprometê-los, visto que as duas unidades pesquisadas exibem eficiência técnica que gravitam em torno de valores das unidades que não participam do aludido programa; em resumo e conforme abordado, a regressão estimada foi: $\mathrm{ET}=\beta_{0}+\beta_{1} \mathrm{GMA}+\beta_{2} \mathrm{GQ}+e$; os resultados obtidos, donde o “*” indica o coeficiente estatisticamente significativo a nível de significância de 5\%, foram:

$$
\begin{aligned}
& \mathrm{ET}=0,454362+0,000018 \mathrm{GMA}-0,119700 \mathrm{GQ} \quad \mathrm{R}^{2}=0,7522 \quad \mathrm{~F}=10,63 \\
& (5,47)^{*} \quad(4,28)^{*} \quad(-0,83) \quad \mathrm{d}=2,65 \quad \mathrm{n}=10
\end{aligned}
$$

Nesta equação inexistem, com significância estatística, autocorrelação dos resíduos, heterocedasticidade e multicolinearidade; verificou-se, além disso, através do teste de Jarque-Bera, que os resíduos também estão normalmente distribuídos; ademais, o valor de F, com nível de significância de 5\%, é estatisticamente significativo; por sua vez, o valor de $\mathrm{R}^{2}$ explica em torno de três quartos das variações da variável dependente e, em particular, a primeira variável independente da Eq. 2 é a única com coeficiente estatisticamente significativo; destarte, os gastos com o meio ambiente contribuem para o incremento da eficiência técnica das unidades investigadas; por seu turno, a participação da empresa no Programa Granja de Qualidade não gera resultados estatisticamente significativos e, por conseguinte, enquanto os benefícios fiscais concedidos pelo fisco estadual às empresas que participam deste programa não estão influenciando a eficiência técnica das unidades, os gastos com a conservação do meio ambiente, representados pela realização e manutenção de lagoa de decantação dos dejetos do processo produtivo da suinocultura, voltados essencialmente para a conservação da fauna, flora, ar, solo, entre outros, estão exercendo efeito direto sobre a eficiência técnica das unidades produtivas.

\section{CONCLUSÕES}

1. Os gastos com o meio ambiente provocaram efeitos positivos sobre os índices de eficiência técnica estimados.

2. A participação no Programa Granja de Qualidade não está influenciando os índices de eficiência técnica das empresas, não obstante a concessão de incentivo fiscal que o programa contempla.

3. A principal motivação para que a participação do número de unidades suinícolas no programa esteja aumentando nos anos recentes, seja representada, majoritariamente, pela obtenção da renúncia fiscal que ele proporciona.

4. A atividade suinícola pode provocar diversos impactos sobre o meio ambiente físico, causando, em conseqüência, múltiplas externalidades negativas e positivas; esses resultados se manifestam no solo, na cobertura vegetal, na águas, no ar, na fauna, na flora e ainda no ambiente socioeconômico.

5. As externalidades negativas podem ser atenuadas ou mitigadas pela realização de gastos com meio ambiente.

\section{LITERATURA CITADA}

Alves, O. A. O estado da arte da suinocultura no estado: Um estudo de caso. Cuiabá: UFMT, 2001. 82p. Monografia Especialização 
Anunciato, K. M. Indicadores de estado na produção suinícola: Um estudo de caso na região do médio-norte do estado de Mato Grosso. Cuiabá: UFMT, 2004. 103p. Dissertação Mestrado

Freitas, E. A. S. F. Valoração contingente de ativos ambientais na suinocultura: Um estudo de caso no município de DiamantinoMT. Cuiabá: UFMT, 2004. 167p. Dissertação Mestrado

Magalhães, K. A.; Campos, R. T. Eficiência técnica e desempenho econômico de produtores de leite no estado do Ceará, Brazil. Revista de Economia e Sociologia Rural, v.44, n.4, p.695711, 2006.

Mariano, J. M. A eficiência dos colonos na agricultura irrigada do Vale do São Francisco: Uma análise comparativa dos modelos de fronteira paramétrica e não paramétrica. Recife: UFPE, 1999. 142p. Tese Doutorado
Mariano, J. M.; Sampaio, Y. A eficiência técnica dos colonos na agricultura irrigada no Vale do São Francisco. Economia Aplicada, v.6, n.2, p.265-285, 2002.

Pereira, B. D. Mato Grosso: Principais eixos viários e a modernização da agricultura. Cuiabá: UFMT, 2007. 55p.

Pereira, B. D.; Mendes, C. M. Eficiência técnica: Arroz sequeiro: Sorriso (MT). Revista de Estudos Sociais, v.4, n.8, p.37-51, 2002.

SEPLAN-MT. Anuário estatístico de Mato Grosso, Cuiabá, 2004. Souza Júnior, J. P. de; Khan, A. S.; Lima, P. V. P. S.; Madrid, R. M. M. Produção de camarão marinho em cativeiro: Uma análise de eficiência técnica, alocativa e de custos. Economia Aplicada, v.9, n.2, p.205-224, 2005.

Timmer, G. P. Using a probabilistic frontier production to measurement technical efficiency. Journal of Political Economic, v.79, n.4, p.776-94, 1971. 